\title{
Personalized Physician Learning Intervention to Improve Hypertension and Lipid Control: Randomized Trial Comparing Two Methods of Physician Profiling
}

\author{
Patrick J. O’Connor, MD, MA, MPH [Senior Clinical Investigator], \\ HealthPartners Institute for Education and Research and HealthPartners Center for Chronic Care \\ Innovation, Minneapolis, Minnesota
}

David J. Magid, MD, MPH [Director of Research for Colorado Permanente Medical Group], Institute for Health Research, Kaiser Permanente, Denver, Colorado

\begin{abstract}
JoAnn M. Sperl-Hillen, MD [Senior Clinical Investigator],
HealthPartners Institute for Education and Research and HealthPartners Center for Chronic Care Innovation, Minneapolis, Minnesota
\end{abstract}

David W. Price, MD [Director of Medical Education for the Colorado Permanente Medical Group],

Institute for Health Research, Kaiser Permanente, Denver, Colorado

\author{
Stephen E. Asche, MA [Manager of Statistical Services], \\ HealthPartners Institute for Education and Research, Minneapolis, Minnesota \\ William A. Rush, PhD [Research Investigator], \\ HealthPartners Institute for Education and Research, Minneapolis, Minnesota \\ Heidi L. Ekstrom, MA [Senior Research Project Manager], \\ HealthPartners Institute for Education and Research, Minneapolis, Minnesota
}

David W. Brand, MSPH [Manager of Research Operations, Senior Project Manager], Institute for Health Research, Kaiser Permanente, Denver, Colorado

Heather M. Tavel, BS [Data Specialist/Programmer],

Institute for Health Research, Kaiser Permanente, Denver, Colorado

Olga V. Godlevsky, BA [Senior Web Developer],

HealthPartners Institute for Education and Research, Minneapolis, Minnesota

Paul E. Johnson, PhD [Professor, Curtis L. Carlson Chair in Decision Sciences], and

Carlson School of Management, University of Minnesota, Minneapolis, Minnesota

\footnotetext{
Corresponding author: Patrick J. O’Connor, MD, MA, MPH, HealthPartners Center for Chronic Care Innovation, 8170 33rd Ave. S., Mail Stop 21111R, Minneapolis, MN 55425, Phone: 952-967-5034, Fax: 952-967-5022, patrick.j.oconnor@ healthpartners.com. Contributorship Statement

Patrick J. O’Connor, David J. Magid, JoAnn M. Sperl-Hillen, David W. Price, Stephen E. Asche, William A. Rush, Heidi L. Ekstrom, David W. Brand, Heather M. Tavel, and Karen Margolis contributed to the planning, conduct and reporting of the work described in the article. Paul E. Johnson contributed to the planning and conduct of the work described in this article. Olga V. Godlevsky contributed to the reporting of the work described in the article.

No conflicts of interest.
} 
Karen L. Margolis, MD, MPH [Senior Clinical Investigator and Director of Clinical Research] HealthPartners Institute for Education and Research and HealthPartners Center for Chronic Care Innovation, Minneapolis, Minnesota

\section{Abstract}

Purpose-To assess the impact of personalized physician learning (PPL) interventions using simulated learning cases on control of hypertension and dyslipidemia in primary care settings.

Methods-A total of 132 primary care physicians, 4,568 eligible patients with uncontrolled hypertension, and 15,392 eligible patients with uncontrolled dyslipidemia were clusterrandomized to one of three conditions: (a) no intervention, (b) PPL-electronic medical record (EMR) intervention in which 12 PPL cases were assigned to each physician based on observed patterns of care in the EMR in the previous year, or (c) PPL-ASSESS intervention in which 12 PPL cases were assigned to each physician based on their performance on four standardized assessment cases. General and generalized linear mixed models were used to account for clustering and to model differences in patient outcomes in the study arms.

Results-Among patients with uncontrolled hypertension at baseline, $49.1 \%, 46.6 \%$, and $47.3 \%$ $(P=0.43)$ achieved BP targets at follow up. Among patients with uncontrolled dyslipidemia at baseline, $37.5 \%, 37.3 \%$, and $38.1 \%(P=0.72)$ achieved low-density lipoprotein cholesterol targets at follow up in PPL-EMR, PPL-ASSESS, and the control group, respectively. Although both systolic blood pressure (BP) $(P<.001)$ and lipid $(P<.001)$ values significantly improved during the study, the group-by-time interaction term showed no differential change in systolic BP values $(P=0.51)$ or lipid values $(P=0.61)$ among the three study arms. No difference in intervention effect was noted when comparing the PPL-EMR with the PPL-ASSESS intervention $(P=0.47)$.

Conclusion-The two PPL interventions tested in this study did not lead to improved control of hypertension or dyslipidemia in primary care clinics during a mean 14-month follow-up period. This null result may have been due in part to substantial overall improvement in BP and lipid control at the study sites during the study.

\section{Keywords}

hypertension; dyslipidemia; primary care; quality improvement; learning intervention; chronic disease

\section{Introduction}

Adequate control of dyslipidemia and hypertension in adults reduces rates of heart attack, stroke, and other adverse health events.(1) The cost of uncontrolled hypertension and dyslipidemia is conservatively estimated to be at least $\$ 100$ billion a year in the United States, and improved control of elevated blood pressure (BP) and dyslipidemia are major national health priorities. $(1,2)$

Delayed initiation or intensification of treatment when patients are not at evidence-based clinical goals is a widely recognized barrier to better hypertension and lipid control.(3-5) Several studies show that, when BP or lipids are not well controlled, the likelihood of 
treatment intensification at a given office visit is about $20 \%-30 \%$, and intensification is often delayed until patients have markedly abnormal BP or lipid values. $(3,4,6)$ Several studies demonstrate that more timely medication intensification improves BP and lipid control, $(7,8)$ and in a previous work we showed that a physician learning intervention based on three standardized simulated learning cases successfully improved glucose control and reduced medication errors in adult patients with type 2 diabetes.(9) In a randomized trial conducted with primary care resident physicians, a standardized set of 12 learning cases in residents successfully improved knowledge of diabetes care and management of standardized diabetes assessment cases.(10) A third trial tested a personalized physician learning (PPL) intervention by randomizing primary care physicians to receive or not receive a personalized set of 12 learning cases (from a library of 480 simulated learning cases) that were targeted to specific deficits in diabetes care identified by analysis of each PCPs EMR-extracted data. This intervention successfully improved glucose control in diabetes patients.(9)

Based on these successful randomized trials in diabetes care, and on the observation that simulated learning interventions of various types have been widely used in many industries and have often been shown to be effective,(11) we decided to extend this intervention strategy to address deficits in BP and lipid control in primary care settings.

\section{Methods}

\section{Study Design and Study Hypotheses}

Figure 1 illustrates the study design, which tests impact of personalized physician learning (PPL) interventions on BP and lipid control, with direct comparison of two distinct methods of assigning personalized learning cases to PCPs. One PCP profiling method assigned a personalized set of 12 learning cases to each PCP based on observed physician-specific patterns of care in EMR data; this intervention arm is referred to as the PPL-EMR study arm. The second PCP profiling method assigned a personalized set of 12 learning cases to each PCP based on PCP performance on a baseline set of 4 assessment cases as described below, and is referred to as the PPL-ASSESS study arm. We hypothesized that both these intervention arms (PPL-EMR and PPL-ASSESS) would be superior to the no-intervention control group, and that the more efficient and scalable PPL-ASSESS intervention would be superior to the PPL-EMR intervention in improving BP and lipid control in primary care. Appendix Figure 1 provides an overview of the study design.

\section{Study Sites and Study Subjects}

This study was conducted at Kaiser Permanente Colorado (KPCO) and at HealthPartners Medical Group (HPMG) in Minnesota. Both are multispecialty practices that provide care to large numbers of adults with hypertension or dyslipidemia, and both use the EpiCare $\odot$ (Verona, WI) EMR system. PCPs provide most hypertension and lipid care at both sites, with referral rates to subspecialists for these problems of less than $5 \%$ per year. The quality of EpiCare laboratory and BP data has been carefully assessed in previous studies, with high correlation between BP values recorded by clinical personnel and lipid values reported from laboratory tests and values extracted from the EMR for analysis.(12),(13) In addition, 
rooming nurses at both sites are trained and periodically retrained on proper BP measurement techniques specified in clinical guidelines.(1) Lipid assays are done in accredited clinical chemistry laboratories using standard assay methods that include both direct and indirect measurement of LDL levels.

To be eligible for this study, a physician had to (a) practice at either HPMG or KPCO, (b) be a general internist or family physician, and (c) provide written informed consent. Criteria for patient inclusion in the analysis were: (a) age 21-75 years on the PCP intervention date, (b) linked to one consented PCP based on EMR designation or visit frequency data, and (c) meet study criteria for uncontrolled BP, uncontrolled LDL, or both.

\section{Randomization}

Eligible PCPs were sent email invitations to participate with a link to an online consent form. Follow-up emails were sent to non-responders twice after the initial invitation email. We identified 173 eligible PCPs at KPCO and 99 at HPMG; 63 at KPCO and 69 at HPMG provided written informed consent. We created an ordered list of consented PCPs within each clinic based on the proportion of their hypertension patients whose hypertension was already under control. We then blocked the PCPs in groups of three and randomly assigned each group to one of the three study arms. When the number of PCPs at a given clinic was not a multiple of 3, we created slightly larger or smaller PCP blocks before randomization.

\section{Description and Implementation of Interventions}

The two PPL interventions developed for this project were based on established learning theory(14-16) and current chronic care delivery models and incorporated current theories of motivation and behavior change.(15-17) In the PPL-EMR study arm, 12 learning cases were assigned to each PCP based on content analysis of deficits in all EMR-recorded care provided by that PCP to patients with hypertension or dyslipidemia in the previous year, as described in Appendix Figure 1. In the PPL-ASSESS study arm, 12 learning cases were assigned based on PCP performance on four standardized assessment cases that targeted specific failures of decision making or knowledge gaps but included no learning feedback. In both intervention arms, the 12 PPL cases selected were personalized to directly address observed deficits in PCP performance.

PCPs in both the PPL-EMR and PPL-ASSESS study arms accessed their personalized and unique set of 12 learning cases on a website with security, monitoring, and tracking functions. PCPs received compensation of $\$ 500$ and as many as 4 hours of American Medical Association category $1 \mathrm{CME}$ credit for completion of all 12 learning cases within 8 weeks after randomization. The date on which the first case was completed is designated the PCP intervention date. The date of the first office visit of an eligible patient with a randomized PCP is designated the index visit date and is required by definition to be after the PCP intervention date.

Learning cases for the PCPs in both the PPL-EMR and PPL-ASSESS intervention arms were assigned from the same overall set of cases designed to cover a wide clinical terrain and be consistent with current evidence-based national and regional guidelines. $(18,19)$ Each 
learning case addressed specific hypertension and lipid knowledge deficits such as tailoring care to patients with selected comorbidities or compelling indications for use of certain classes of antihypertensive or lipid medications (Appendix Figure 1). PCPs managed their personalized set of 12 learning cases until they achieved mastery, which was defined as achieving BP and lipid targets within 6 months of simulated time with no risky prescribing events. In each learning case, PCPs received learning feedback based on their clinical moves in the learning cases. Learning feedback provided after each simulated encounter (mean number of encounters, six per simulated case) addressed appropriate initiation or adjustment of preferred drugs, assessment of adherence, appropriate visit interval, ordering of safety labs, nutrition and exercise recommendations, appropriate use of consultants, and avoidance of potentially dangerous drug-drug or drug-condition interactions (see screen shot in Appendix Figure 2). Feedback was also given to reinforce effective care practices. Each of the 12 learning cases took about 15 minutes to complete.

\section{Definition of Key Variables}

Hypertension Control-To be included in the hypertension analyses reported here, study-eligible patients had to have hypertension in the year before the PCP randomization date by meeting at least one of these criteria: (a) Two or more ICD-9 codes for hypertension on two or more dates, (b) one ICD-9 diagnostic code for hypertension plus a filled prescription for a hypertension medication, (c) two consecutive elevated BP values, or (d) one ICD-9 diagnostic code and one elevated BP. In addition, to be included in the hypertension analyses presented here, patients had to have uncontrolled hypertension (BP $>=140 / 90 \mathrm{~mm} \mathrm{Hg}$ or $\mathrm{BP}>=130 / 80 \mathrm{~mm} \mathrm{Hg}$ for patients with diabetes) at their index visit and at the visit immediately before the index visit. Follow-up hypertension control was based on the mean of the last two systolic BP and diastolic BP values in the postintervention period. Mean change in systolic and diastolic BP values were also evaluated.

Lipid Control—To be included in the dyslipidemia analyses reported here, study-eligible patients had to meet at least one of these criteria in the year before the randomization date: (a) any LDL value > $=70 \mathrm{mg} / \mathrm{dL}$; (b) assigned one of more of these ICD-9 dyslipidemia diagnosis codes: 272.0, 272.2, 272.4; or (c) any medication order for a statin. From this pool of patients, we included in the analysis only those whose most recent LDL value before the index visit was above goal, defined in waterfall fashion as $<70 \mathrm{mg} / \mathrm{dL}$ for patients with coronary heart disease (CHD), $<100 \mathrm{mg} / \mathrm{dL}$ for remaining patients with diabetes, $<130$ $\mathrm{mg} / \mathrm{dL}$ for remaining patients with two or more major cardiovascular risk factors (hypertension, smoking, or BMI $>25 \mathrm{~kg} / \mathrm{m}^{2}$ ), and $<160 \mathrm{mg} / \mathrm{dL}$ for all others.(18) Postintervention lipid control was based on the last LDL in the post-intervention period. Mean change in LDL values was also examined.

Patient Variables-Patient age, sex, race/ethnicity, vital signs, height, weight, smoking status, diagnosis data, and laboratory test results were extracted from the EMR. The validity of these EMR-derived data has been carefully assessed in previous studies. $(12,13)$

Identification of Diabetes and CHD-Accurate classification of diabetes and CHD status in the 2-year period before the randomization date was important because patients 
with these conditions had condition-specific BP or LDL goals during the study period. Patients were classified as having diabetes if, in the 2 years before the index visit date, they had (a) two or more outpatient ICD-9 codes for diabetes (250.xx), (b) a filled prescription for a diabetes-specific medication such as insulin, sulfonylurea, or meglitamides, or (c) one outpatient ICD-9 diabetes code plus a filled prescription for metformin or a thiazolidinedione. Patients were classified as having CHD if they had one inpatient or two or more outpatient ICD-9 codes 410-414 or 429.2. The diabetes identification method has an estimated sensitivity of 0.91 and a positive predictive value of 0.94; the CHD identification method has an estimated sensitivity of 0.89 and a positive predictive value of 0.79.(20)

\section{Analytic Approach}

Blood pressure and lipid control were analyzed using generalized linear mixed models (logit link) predicting post-intervention BP or lipid control from study arm, a fixed effect for site, and a random effect for provider because providers were the unit of randomization. Likelihood ratio tests comparing the model with and without the two degrees of freedom (df) study arm contrast were computed as the key test of a study arm effect. Pairwise comparisons of study arms tested the parameter for study arm in each of the three individual regression equations and whether these parameters were significantly different from zero (eg, parameter estimate/SE >2.0).

Analyses of change in systolic BP, diastolic BP, and LDL follow a similar approach but use general linear mixed models with fixed effects of study arm, time (baseline or postintervention), interaction of study arm and time, a fixed effect for site, and a random effect for provider. A significant $(P<.05)$ result for the study-arm-by-time interaction term indicates differential change by study arm over time.

Sensitivity analysis of the BP control and BP change outcomes included an examination of patients having visits with providers in only one study arm ( $89 \%$ of patients from the main analysis) and an analysis examining the mean of the first two post-intervention BP values rather than the last two post-intervention $\mathrm{BP}$ values from the main analysis.

The study was planned to have $80 \%$ power (alpha $=0.05 / 3=.017$, two-sided test) to detect a difference in BP control at follow up of $20 \%$ in the control arm versus $27 \%$ in the PPLASSESS group, assuming 40 providers per arm, 30 uncontrolled hypertension patients per provider, and a provider-level ICC of .015 for BP control. In a post-hoc power analysis for the lipid control analysis, the study had $80 \%$ power (alpha $=0.05 / 3=.017$, two-sided test) to detect a difference in follow-up LDL control of $35.0 \%$ in the control arm versus $38.4 \%$ in the PPL-ASSESS group, using observed values of 130 providers, 118 uncontrolled lipid patients per provider, and a provider-level ICC of .005 for LDL control.

\section{Protection of Human Study Subjects and Role of the Funder}

This study was reviewed in advance, approved, and monitored by the HealthPartners Institutional Review Board (IRB) and the Kaiser Permanente of Colorado IRB. Patient informed consent was waived by both IRBs. This randomized trial is registered with clinicaltrials.gov, NCT00903071. 


\section{Results}

Figure 2 is a Consort diagram that describes selection and follow-through of eligible PCPs and patient study subjects. A total of 130 eligible PCPs and their patients with baseline uncontrolled hypertension $(n=4,568)$ were included in the BP analysis, and the same 130 PCPs and their patients with baseline uncontrolled LDL $(\mathrm{n}=15,392)$ were included in the LDL analysis.

Table 1 shows characteristics of patients included in the hypertension and lipid analysis. The analytic sample $(n=4,568)$ includes patients with uncontrolled hypertension $(\mathrm{BP}>=140 / 90$ $\mathrm{mm} \mathrm{Hg}$ or $\mathrm{BP}>=130 / 80 \mathrm{~mm} \mathrm{Hg}$ for patients with diabetes) at their index visit and at the visit immediately before the index visit and two or more follow-up BP measurements, the last of which was taken at least 4 months after the index visit. Study providers were linked to a median of 27 (range, 5-134) patients with uncontrolled BP. Baseline levels of adequate hypertension control were relatively high at baseline among the 37,755 individuals with any indication of hypertension at baseline who made up the initial study pool. BP control at baseline was defined as the mean of the index visit BP and previous visit BP of $<140 / 90 \mathrm{~mm}$ $\mathrm{Hg}$ (or $<130 / 80 \mathrm{~mm} \mathrm{Hg}$ for patients with diabetes); BP control was $82 \%$ at baseline at one site and $75 \%$ at the other.

The analytic sample for the lipid analysis $(n=15,392)$ includes patients with uncontrolled LDL (see Lipid Control under Definition of Key Variables) at baseline and a postintervention LDL value. Study providers were linked to a median of 117 (range, 20-270) patients with uncontrolled LDL. Baseline levels of adequate lipid control among the initial sample pool of 91,680 with any LDL value $>=70 \mathrm{mg} / \mathrm{dL}$, an ICD-9 diagnosis code (272.0, $272.2,272.4$ ), or any medication order for a statin were $22 \%$ for one site and $24 \%$ for the other.

Among the 130 study-consented PCPs linked to patients in Table 1, 68 were from site 1 and 62 from site 2 . The mean age of PCPs was 48.5 years $(\mathrm{SD}=8.6), 50.0 \%$ were family practice physicians, and $56.2 \%$ were female. The mean days worked per week was $4.0(\mathrm{SD}=0.8)$ and the mean years since graduation was $21.2(\mathrm{SD}=8.8)$. All 4 assessment cases were completed by $82.8 \%$, and all 12 learning cases were completed by $79.3 \%$ of PCPs in the two intervention arms. The mean number of the 12 learning cases completed was 9.7 in the PPLEMR intervention arm and 9.9 in the PPL-ASSESS intervention arm. There were no statistically significant differences in PCPs on these attributes by study arm.

Table 2 shows changes in BP control and in systolic BP and diastolic BP values among eligible patients with uncontrolled hypertension at baseline. There was significant improvement in BP control in all three study arms over time, with post-intervention BP controlled in $47 \%$ of those whose hypertension was uncontrolled at baseline. Both systolic and diastolic BP improved in all study arms, with a mean reduction of $11.0 \mathrm{~mm} \mathrm{Hg}$ for systolic BP and $5.4 \mathrm{~mm} \mathrm{Hg}$ for diastolic BP over 13.2 months. However, there were no significant differences in BP control or changes in systolic BP or diastolic BP in patients exposed to either PPL-EMR or PPL-ASSESS relative to the no-intervention control group. 
Table 3 shows there were no clinically significant pairwise differences in SBP or DBP levels or BP control across the three study arms.

Secondary analyses were done to assess intervention effects after excluding patients whose data may have been "contaminated" by care received during the follow-up period from PCPs assigned to different study arms. Results of these analyses were very similar to those obtained when analyzing all patients, with no statistically significant differences across study arms. Secondary analyses were also done using the first two post-index BP measures rather than the last two post-index BP measures. This analysis showed a mean reduction in systolic BP of $9.2 \mathrm{~mm} \mathrm{Hg}$ and a mean reduction in diastolic BP of $4.2 \mathrm{~mm} \mathrm{Hg}$ over a mean of 4.0 months, with no significant differences among study arms.

Table 2 also shows that LDL levels improved over time in all three study arms, with a mean LDL reduction of $18.1 \mathrm{mg} / \mathrm{dL}$ between baseline and follow-up LDL measures over a mean follow-up period of 15.1 months, with no significant differences in LDL reduction or proportion of subjects at LDL goal in the three study arms. Table 3 shows there were no significant pairwise differences in LDL levels of LDL control across the three study arms.

The proportion of adults with uncontrolled hypertension who had active management of their hypertension medications during the post-intervention period in the PPL-EMR, PPLASSESS and no-intervention control group was 57\%, 56\%, and 53\% at Site 1 and was 57\%, $68 \%$, and $70 \%$ at Site 2, indicating that neither intervention led to a significant increase in active management of hypertension medications at either study site.

\section{Discussion}

The objective of this project was to assess the ability of two personalized physician learning (PPL) interventions to improve hypertension and lipid control in primary care practice. The study was conducted in community-based primary care clinics and was designed to minimize selection effects by having broad patient eligibility criteria and waiving written informed consent for patients. However, although prior randomized studies showed benefits on glucose control in diabetes care, neither of the two PPL interventions tested was able to improve hypertension or lipid control rates compared with a no-intervention control group.

Several factors may have contributed to our null result. First, it may be that PCP knowledge gaps about hypertension and lipid care are smaller than knowledge gaps about glucose control. If so, learning intervention such as PPL that targets knowledge deficits may be more effective for the more clinically complex domains such as glucose control, and less effective for more straightforward clinical domains such as BP and lipid control. Second, it may be that the high baseline rate of hypertension control and observed strong overall improvement trends in both hypertension control and lipid control during the study period contributed to the null findings. Finally, about $20 \%$ of intervention group PCPs did not complete their set of 12 PPL cases, which may have weakened the intervention effect. However, similar rates of intervention completion were observed in previous successful PPL interventions.

A number of factors limit the interpretation of our data. First, the study was conducted at only two medical groups, and results may not be generalizable to other care settings or 
patient populations. In particular, it is not known if such an intervention would improve care in lower-performing care systems or medical groups. Second, the inaccuracies of officederived BP measurements are well known.(21) However, the randomized study design and comparison before and after BP data for each patient are design features that may somewhat mitigate the impact of potentially imprecise clinic-based BP measures. Moreover, we conducted the study at sites where nursing staff are trained and periodically retrained on proper BP measurement according to a specified protocol.(22) As digital BP measurement devices come into wider use, the accuracy of clinic BP measurements may improve over time. Third, our study design did not permit assessment of joint effects on care quality when PPL interventions are deployed in tandem with other types of care-improvement interventions. In particular, both medical groups had sophisticated EHR systems and were developing other approaches such as pharmacist case management for some hypertension patients during the study period.

In summary, this PPL intervention did not lead to significant improvements in either BP or lipid control at these relatively high-performing study sites. The impact of learning interventions on medical groups or care systems with poorer baseline BP control remains to be seen, but beneficial impact may be limited if knowledge deficits are not a major factor contributing to suboptimal care. It is possible that more effective physician learning interventions for BP or lipid control may be designed in the future. Until such studies are conducted, our results suggest that a personalized physician learning intervention may not be as effective as other strategies such as pharmacist case management, point-of-care EMRbased clinical decision support, or patient incentives as a strategy to successfully improve adult hypertension or lipid control in primary care settings.(23-26)

\section{Supplementary Material}

Refer to Web version on PubMed Central for supplementary material.

\section{Acknowledgments}

Funding

This study was funded by the National Heart, Lung, and Blood Institute at the National Institutes of Health Department of Health and Human Services 1RO1HL089451 to HealthPartners Institute for Education and Research (formerly HealthPartners Research Foundation), Patrick O'Connor MD MA MPH, Principal Investigator.

\section{References}

1. Chobanian AV, Bakris GL, Black HR, et al. Seventh Report of the Joint National Committee on Prevention, Detection, Evaluation, and Treatment of High Blood Pressure. Hypertension. 2003; 42(6):1206-52. [PubMed: 14656957]

2. Paramore LC, Halpern MT, Lapuerta $P$, et al. Impact of poorly controlled hypertension on healthcare resource utilization and cost. Am J Manag Care. 2001; 7(4):389-98. [PubMed: 11310193]

3. Fine LJ, Cutler JA. Hypertension and the treating physician: understanding and reducing therapeutic inertia. Hypertension. 2006; 47(3):319-20. [PubMed: 16432049]

4. Berlowitz DR, Ash AS, Hickey EC, et al. Inadequate management of blood pressure in a hypertensive population. N Engl J Med. 1998; 339(27):1957-63. [PubMed: 9869666] 
5. O'Connor PJ. Overcome clinical inertia to control systolic blood pressure. Arch Intern Med. 2003; 163(22):2677-8. [PubMed: 14662620]

6. O'Connor, PJ.; Sperl-Hillen, JM.; Johnson, PE., et al. Clinical Inertia and Outpatient Medical Errors. In: Henriksen, K.; Battles, J.; Lewin, D., et al., editors. Advances in Patient Safety: From Research to Implementation. Rockville, MD: Agency for Healthcare Research and Quality (AHRQ); 2005. p. 293-308.

7. Pyorala K, Pedersen TR, Kjekshus J, et al. Cholesterol lowering with simvastatin improves prognosis of diabetic patients with coronary heart disease. A subgroup analysis of the Scandinavian Simvastatin Survival Study (4S). Diabetes Care. 1997; 20(4):614-20. [PubMed: 9096989]

8. O'Connor PJ, Vazquez-Benitez G, Schmittdiel JA, et al. Benefits of early hypertension control on cardiovascular outcomes in patients with diabetes. Diabetes Care. 2013; 36(2):322-7. [PubMed: 22966094]

9. Sperl-Hillen JM, O'Connor PJ, Rush WA, et al. Simulated physician learning program improves glucose control in adults with diabetes. Diabetes Care. 2010; 33(8):1727-33. [PubMed: 20668151]

10. Sperl-Hillen JM, O'Connor P, Ekstrom HL, et al. Using Simulation Technology to Teach Diabetes Care Management Skills to Resident Physicians. J Diabetes Sci Technol. 2013; 7(5):1243-54. [PubMed: 24124951]

11. Yesavage JA, Jo B, Adamson MM, et al. Initial cognitive performance predicts longitudinal aviator performance. J Gerontol B Psychol Sci Soc Sci. 2011; 66(4):444-53. [PubMed: 21586627]

12. Daugherty SL, Masoudi FA, Ellis JL, et al. Age-dependent gender differences in hypertension management. J Hypertens. 2011; 29(5):1005-11. [PubMed: 21330934]

13. Maddox TM, Ross C, Tavel HM, et al. Blood Pressure Trajectories and Associations With Treatment Intensification, Medication Adherence, and Outcomes Among Newly Diagnosed Coronary Artery Disease Patients. Circ Cardiovasc Qual Outcomes. 2010; 3(4):347-57. [PubMed: 20488918]

14. Bainbridge, L. The process controller. In: Singleton, WT., editor. The Anlaysis of Practical Skills. Baltimore, MD: University Park Press; 1978. p. 236-63.

15. Pine, BJI. Mass Customization. Boston, MA: Harvard Business School Press; 1999. Masscustomizing products and services; p. 171-212.

16. Schafer, J.; Konstan, J.; Reidl, J., editors. Recommender systems in e-commerce; ACM on Electronic Conference; Association for Computing Machinery; 1999.

17. Johnson PE, Veazie PJ, Kochevar L, et al. Understanding variation in chronic disease outcomes. Health Care Manag Sci. 2002; 5:175-89. [PubMed: 12363045]

18. NCEP. Third Report of the National Cholesterol Education Program (NCEP) Expert Panel on Detection, Evaluation, and Treatment of High Blood Cholesterol in Adults (Adult Treatment Panel III) final report. Circulation. 2002; 106(25):3143-421. [PubMed: 12485966]

19. Grundy SM, Cleeman JI, Merz CN, et al. Implications of recent clinical trials for the National Cholesterol Education Program Adult Treatment Panel III guidelines. Circulation. 2004; 110(2): 227-39. [PubMed: 15249516]

20. O'Connor PJ, Rush WA, Pronk NP, et al. Identifying diabetes mellitus or heart disease among health maintenance organization members: sensitivity, specificity, predictive value, and cost of survey and database methods. Am J Manag Care. 1998; 4(3):335-42. [PubMed: 10178496]

21. Jones DW, Appel LJ, Sheps SG, et al. Measuring blood pressure accurately: New and persistent challenges. JAMA. 2003; 289(8):1027-30. [PubMed: 12597757]

22. Institue for Clinical Systems Improvement (ICSI). Health Care Guideline. Bloomington, MN: ICSI; 2005 Oct. Health care guideline: Hypertension diagnosis and treatment. Report No

23. Margolis KL, Asche SE, Bergdall AR, et al. Effect of home blood pressure telemonitoring and pharmacist management on blood pressure control: a cluster randomized clinical trial. JAMA. 2013; 310(1):46-56. [PubMed: 23821088]

24. Green BB, Cook AJ, Ralston JD, et al. Effectiveness of home blood pressure monitoring, Web communication, and pharmacist care on hypertension control: a randomized controlled trial. JAMA. 2008; 299(24):2857-67. [PubMed: 18577730] 
25. Bardach NS, Wang JJ, De Leon SF, et al. Effect of pay-for-performance incentives on quality of care in small practices with electronic health records: a randomized trial. JAMA. 2013; 310(10): 1051-9. [PubMed: 24026600]

26. Petersen LA, Simpson K, Pietz K, et al. Effects of individual physician-level and practice-level financial incentives on hypertension care: a randomized trial. JAMA. 2013; 310(10):1042-50. [PubMed: 24026599] 


\section{Step 1 \\ Baseline \\ assessment of PCP \\ learning deficits \\ Step 2 \\ Randomization \\ of PCPs}

Step 3

Assignment

of learning

cases

\section{Step 4}

Completion of

learning

intervention

\section{Step 5}

Analysis:

Identify patients

exposed to study

PCPs

Step 6

Analysis:

Assess clinical

outcomes of eligible

patients

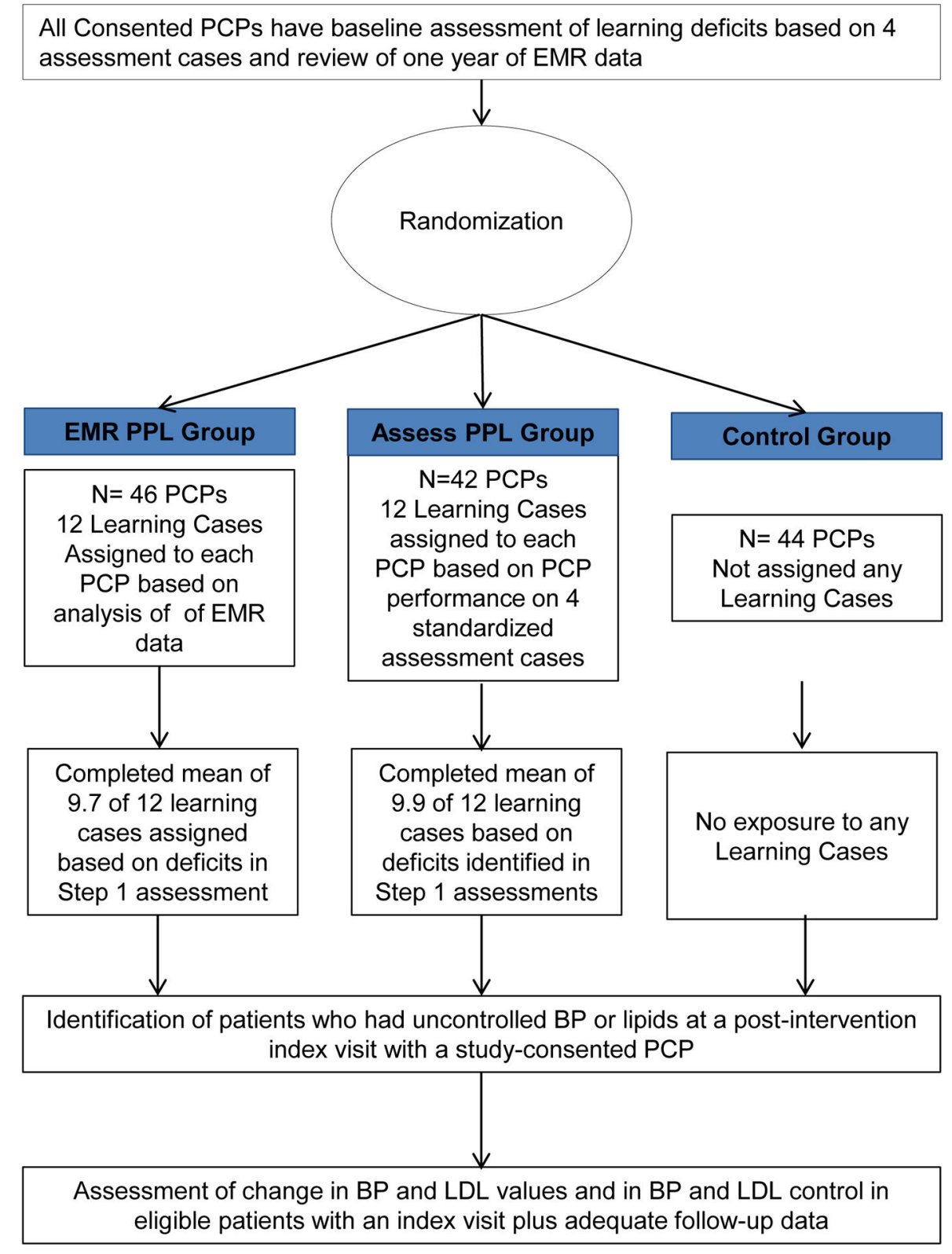

All Consented PCPs have baseline assessment of learning deficits based on 4 assessment cases and review of one year of EMR data

Figure 1.

Study design with description of personalized physician learning (PPL) intervention 


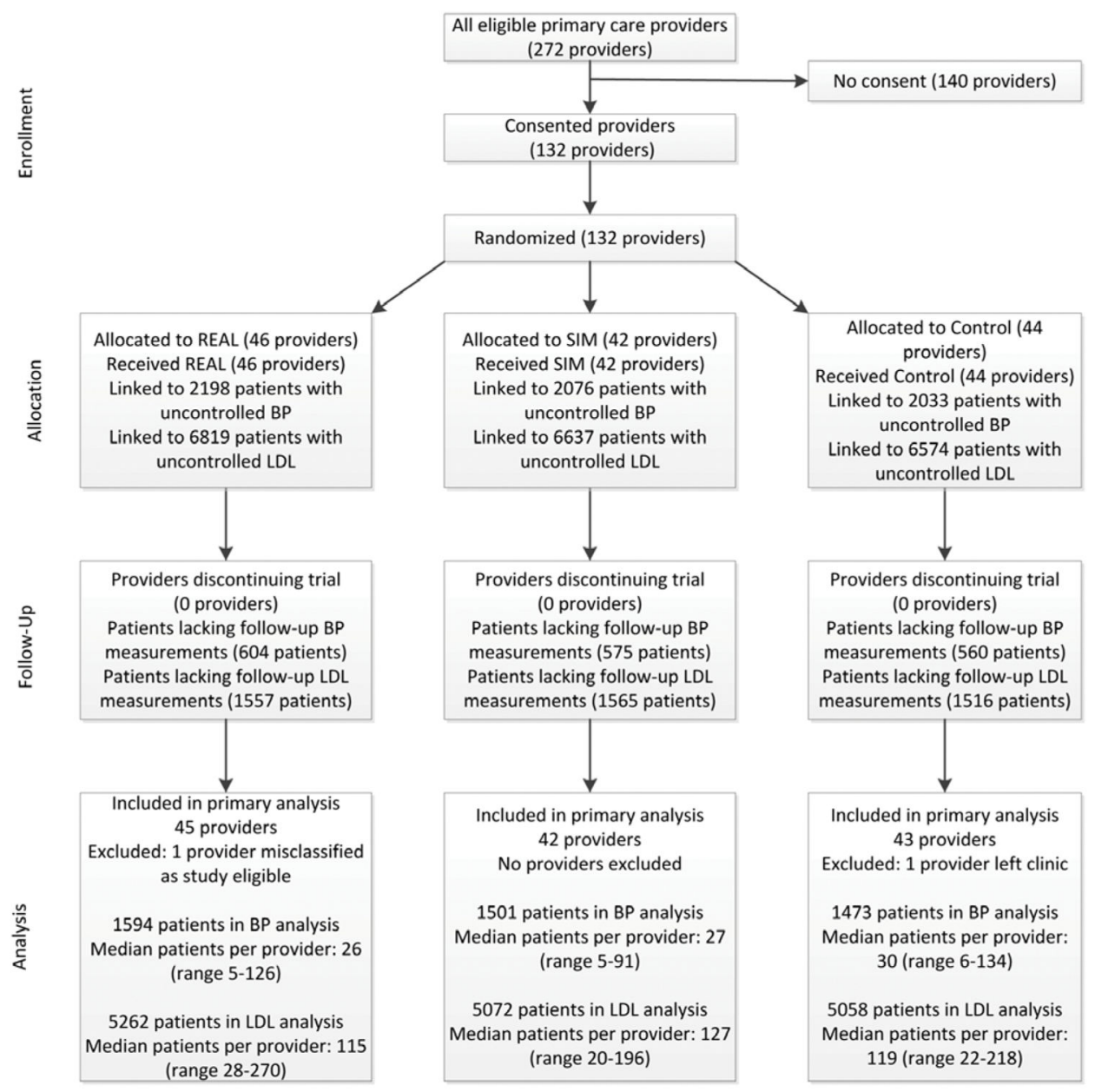

Figure 2.

Consort diagram showing identification and follow-through of potentially eligible clinics, primary care providers, and patient study subjects in the three randomized study arms. 


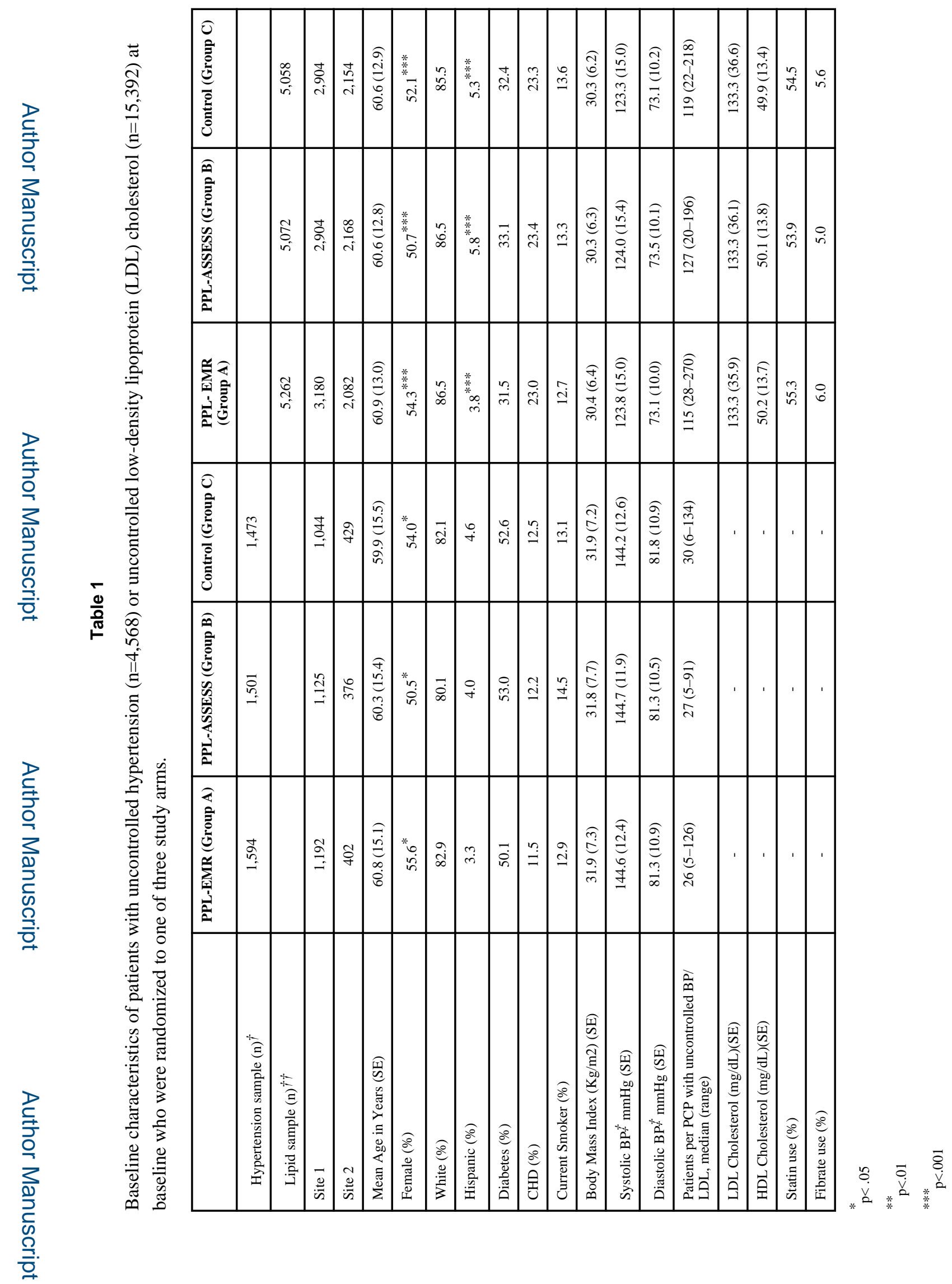

BMJ Qual Saf. Author manuscript; available in PMC 2015 December 01. 


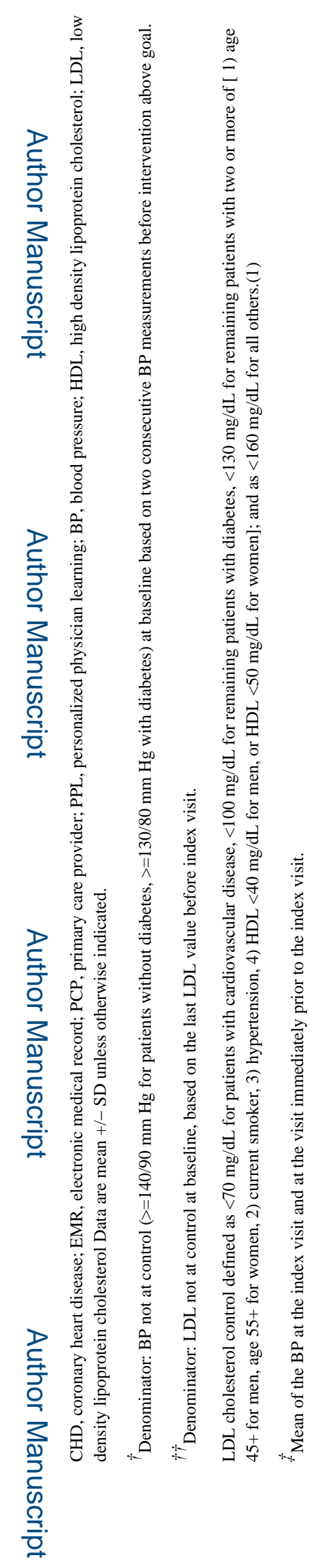

BMJ Qual Saf. Author manuscript; available in PMC 2015 December 01. 


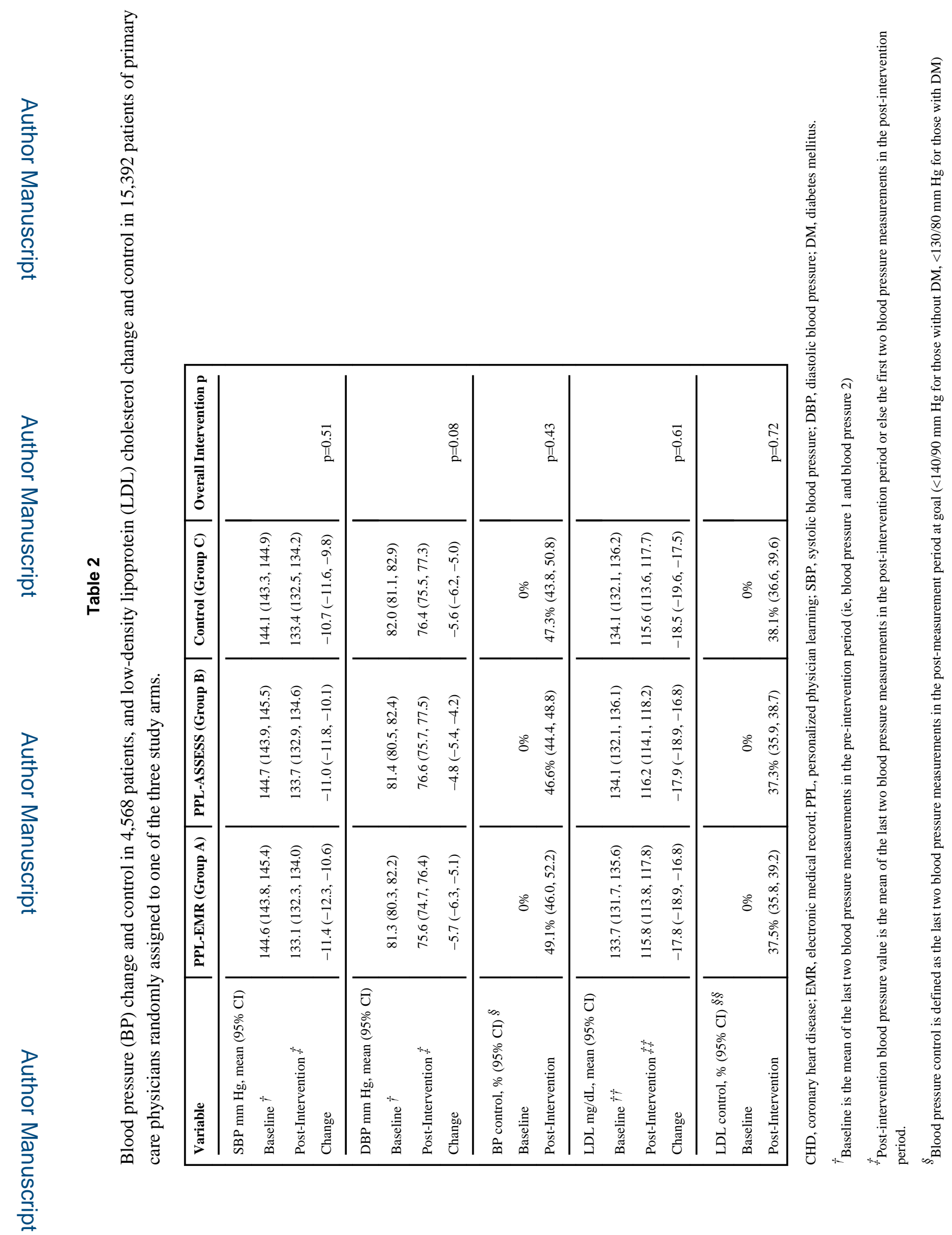

BMJ Qual Saf. Author manuscript; available in PMC 2015 December 01. 


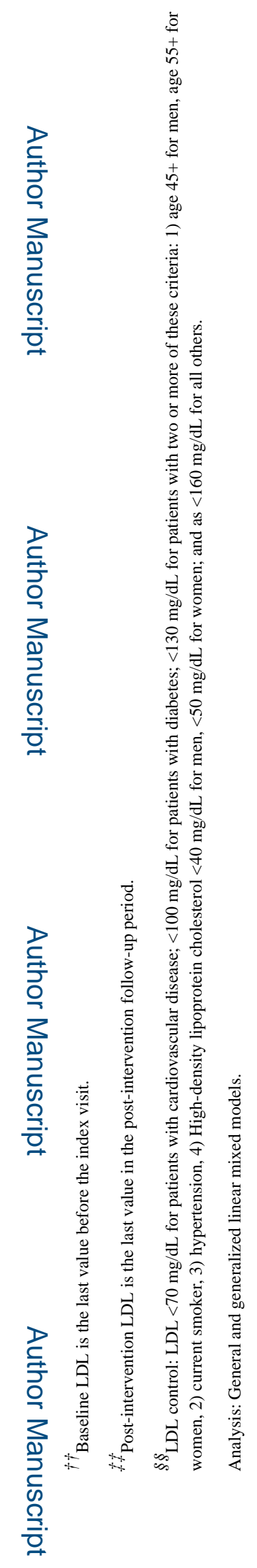

BMJ Qual Saf. Author manuscript; available in PMC 2015 December 01. 


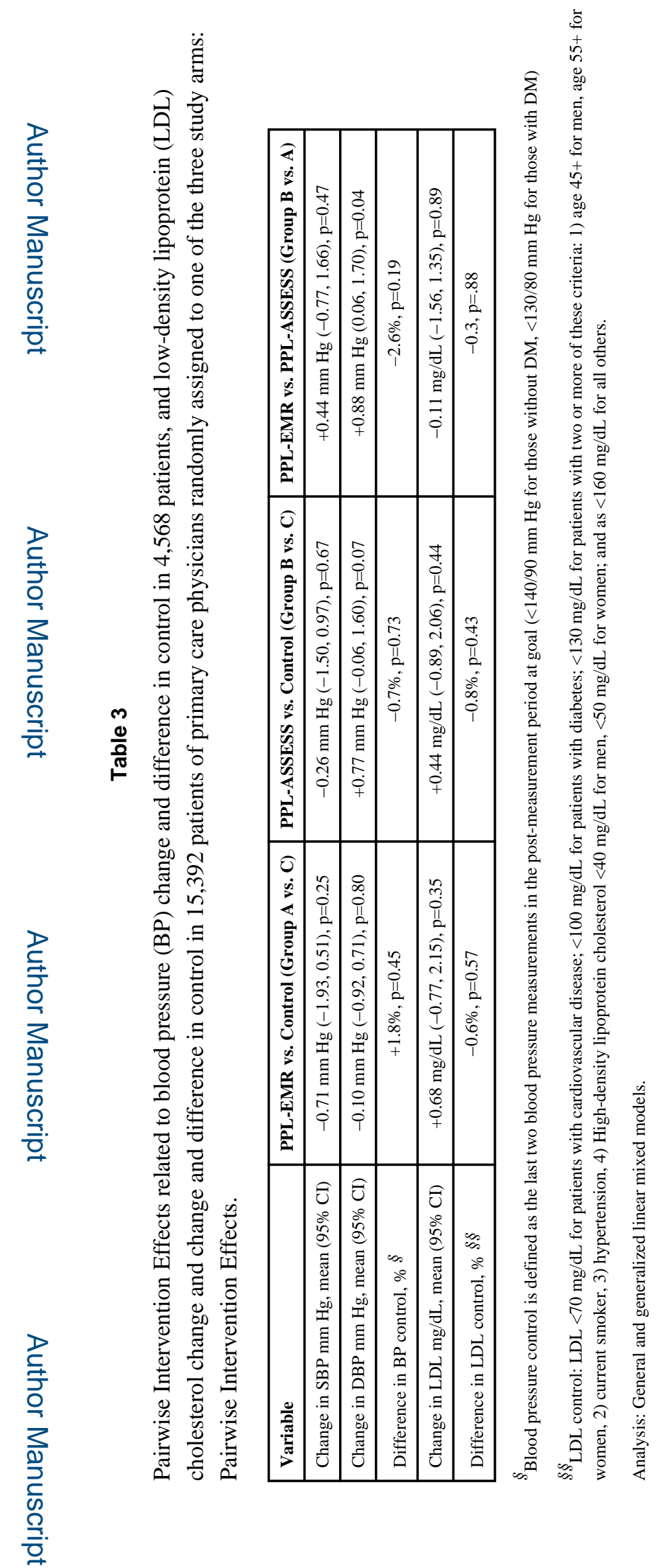

BMJ Qual Saf. Author manuscript; available in PMC 2015 December 01. 